\title{
Computation and interpretation of molecular Omega intracules
}

\author{
Deborah L. Crittenden and Peter M. W. Gill \\ Research School of Chemistry, Australian National University, Australian Capital Territory 0200, Australia
}

(Received 19 February 2007; accepted 9 May 2007; published online 2 July 2007)

\begin{abstract}
The Omega intracule is a three-dimensional function that describes the relative positions, momenta, and directions of motion of pairs of electrons in a system. In this paper, we describe the computation of the Omega intracule for a molecular system whose electronic wave function is expanded in a Gaussian basis set. This is followed by implementation details and numerical tests. Finally, we use the Omega intracules of a number of small systems to illustrate the power of this function to extract simple physical insights from complicated wave functions. (C) 2007 American Institute of Physics.
\end{abstract}

[DOI: $10.1063 / 1.2746028$ ]

\section{INTRODUCTION}

The electronic wave function of a molecular system is a complicated multidimensional object, depending explicitly on the coordinates of all the electrons and parametrically on the positions of the nuclei, and this makes it difficult to interpret and impossible to visualize. Extracting reduceddimension functions that contain the key physical information from a molecular electronic wave function is desirable for two reasons. First, such functions can be easily visualized, thus giving an intuitive understanding of the behavior of the electrons. Second, they may provide a foundation for the development of new approaches to electronic structure theory.

The first reduced-dimension function constructed from an electronic wave function was the one-electron density, $\rho(\mathbf{r})$. This three-dimensional function is easily visualized and, upon analysis, yields information about chemical bonding in a system, according to the local buildup or depletion of electron density. Following the discovery of the Hohenberg-Kohn theorem, ${ }^{1}$ which states that the exact energy of the ground state of a system is a unique functional of the exact electronic density, $\rho(\mathbf{r})$ has been used as the foundation of an entire class of models for calculating electron correlation energies, the density functional theories (DFTs). A large number of density functionals have been developed and tested, and DFT is now a well-established paradigm within the quantum chemistry community.

Subsequently, $\mathrm{we}^{2-10}$ and others ${ }^{11-52}$ have investigated reduced-dimension functions that retain explicit two-electron information. The most familiar of these functions are the two-electron position density $\rho\left(\mathbf{r}_{1}, \mathbf{r}_{2}\right)$ and the two-electron momentum density $\pi\left(\mathbf{p}_{1}, \mathbf{p}_{2}\right)$. These can be reduced further to give the position intracule $P(u)$, which is the probability density for the relative distance $u=\left|\mathbf{r}_{1}-\mathbf{r}_{2}\right|$, and the momentum intracule $M(v)$, which is the probability density for the relative momentum $v=\left|\mathbf{p}_{1}-\mathbf{p}_{2}\right|$. Such intracules are much simpler to visualize and interpret than their parent twoelectron probability densities.

Recently, we have introduced a family of intracules that contain information about both the relative positions of the electrons and their relative momenta. ${ }^{5-8}$ The Omega intrac- ule $\Omega(u, v, \omega)$ measures the probability that two electrons will be found at a distance $u$, moving with relative momentum $v$, and with a dynamical angle $\omega$ where $\mathbf{u} \cdot \mathbf{v}=u v \cos \omega$. The Wigner intracule $W(u, v)$ and Angle intracule $Y(\omega)$ can be obtained by integrating $\Omega(u, v, \omega)$ over $\omega$ or over $u$ and $v$, respectively.

Although the Omega and Wigner intracules are not true probability densities, for the existence of a wave function in phase space is forbidden by the Heisenberg uncertainty principle, they possess many of the mathematical properties of a true probability density. We have therefore postulated that these functions may be used as the basis of a new type of electronic structure model, in which the correlation energy is obtained by contracting the intracule with an appropriate correlation kernel.

In previous work, we have found closed-form expressions for the Omega and Wigner intracules in atoms and linear molecules. ${ }^{8}$ Through these, the variables $u$ and $v$ have been relatively well studied, and both their physical meaning and their importance in describing electron correlation are becoming increasingly clear. In fact, we have demonstrated that Wigner intracule functional theories have the potential to estimate atomic correlation energies both accurately and efficiently. ${ }^{8}$

The aim of the present work is to extend this approach by giving details of the derivation and computation of Omega intracules for more general molecular systems. Then, by constructing and examining Angle intracules for a range of atoms and molecules, we seek to develop an understanding of the physical significance of the angle $\omega$. Atomic units (a.u.) are used throughout.

\section{THEORY}

\section{A. The $\Omega(u, v, \omega)$ intracule}

The ideal starting point for deriving a phase-space intracule would be a phase-space wave function but, although position-space and momentum-space wave functions are well defined, the Heisenberg uncertainty principle forbids the construction of one in phase space. Therefore, we turn instead to the Wigner distribution, ${ }^{53}$ 


$$
\begin{aligned}
W\left(\mathbf{r}_{1}, \ldots, \mathbf{r}_{\mathbf{n}}, \mathbf{p}_{1}, \ldots, \mathbf{p}_{\mathbf{n}}\right) & \\
= & \frac{1}{\pi^{3 n}} \int \Psi\left(\mathbf{r}_{1}+\mathbf{q}_{1}, \ldots, \mathbf{r}_{\mathbf{n}}+\mathbf{q}_{\mathbf{n}}\right)^{*} \Psi\left(\mathbf{r}_{\mathbf{1}}-\mathbf{q}_{\mathbf{1}}, \ldots, \mathbf{r}_{\mathbf{n}}-\mathbf{q}_{\mathbf{n}}\right) \\
& \times e^{2 i\left(\mathbf{p}_{\mathbf{1}} \cdot \mathbf{q}_{1}+\cdots+\mathbf{p}_{\mathbf{n}} \cdot \mathbf{q}_{\mathbf{n}}\right)} d \mathbf{q}_{\mathbf{1}} \cdots d \mathbf{q}_{\mathbf{n}},
\end{aligned}
$$

which is a quantum phase-space density that possesses many of the mathematical properties of a probability density but which is not necessarily everywhere positive.

Because we seek two-electron information, we integrate over the variables $\mathbf{r}_{\mathbf{3}}$ to $\mathbf{r}_{\mathbf{n}}$ and $\mathbf{p}_{\mathbf{3}}$ to $\mathbf{p}_{\mathbf{n}}$ to obtain the secondorder reduced Wigner distribution,

$$
\begin{aligned}
W_{2}\left(\mathbf{r}_{1}, \mathbf{r}_{2}, \mathbf{p}_{1}, \mathbf{p}_{2}\right)= & \frac{1}{\pi^{6}} \int \rho_{2}\left(\mathbf{r}_{1}+\mathbf{q}_{1}, \mathbf{r}_{1}-\mathbf{q}_{1}, \mathbf{r}_{2}+\mathbf{q}_{2}, \mathbf{r}_{2}-\mathbf{q}_{2}\right) \\
& \times e^{2 i\left(\mathbf{p}_{1} \cdot \mathbf{q}_{1}+\mathbf{p}_{2} \cdot \mathbf{q}_{2}\right)} d \mathbf{q}_{1} d \mathbf{q}_{2}
\end{aligned}
$$

where $\rho_{2}\left(\mathbf{r}_{1}^{\prime}, \mathbf{r}_{2}^{\prime}, \mathbf{r}_{1}, \mathbf{r}_{2}\right)$ is the spinless reduced second-order density matrix ${ }^{54}$ of the wave function.

The Omega intracule can then be extracted from $W_{2}$ by using Dirac delta functions to pick out the desired values of $u, v$, and $\omega$ and integrating to obtain

$$
\begin{aligned}
\Omega(u, v, \omega)= & \int W_{2}\left(\mathbf{r}_{1}, \mathbf{r}_{2}, \mathbf{p}_{1}, \mathbf{p}_{2}\right) \delta\left(r_{12}-u\right) \delta\left(p_{12}-v\right) \\
& \times \delta\left(\theta_{u v}-\omega\right) d \mathbf{r}_{1} d \mathbf{r}_{2} d \mathbf{p}_{1} d \mathbf{p}_{2} \\
= & \frac{1}{8 \pi^{3}} \int \rho_{2}(\mathbf{r}, \mathbf{r}+\mathbf{q}, \mathbf{r}+\mathbf{u}+\mathbf{q}, \mathbf{r}+\mathbf{u}) e^{i \mathbf{v} \cdot \mathbf{q}} \\
& \times \delta\left(\theta_{u v}-\omega\right) d \mathbf{r} d \mathbf{q} d \mathbf{\Omega}_{\mathbf{u}} d \mathbf{\Omega}_{\mathbf{v}} \\
= & \frac{1}{8 \pi^{3}} \int \rho_{2}\left(\mathbf{r}_{1}, \mathbf{r}_{2}, \mathbf{r}_{2}+\mathbf{u}, \mathbf{r}_{\mathbf{1}}+\mathbf{u}\right) e^{i \mathbf{v} \cdot\left(\mathbf{r}_{2}-\mathbf{r}_{1}\right)} \\
& \times \delta\left(\theta_{u v}-\omega\right) d \mathbf{r}_{1} d \mathbf{r}_{2} d \mathbf{\Omega}_{\mathbf{u}} d \mathbf{\Omega}_{\mathbf{v}} .
\end{aligned}
$$

If the wave function is expanded in a basis of oneelectron functions $\phi_{a}(\mathbf{r})$, we have

$$
\rho_{2}\left(\mathbf{r}_{\mathbf{1}}^{\prime}, \mathbf{r}_{\mathbf{2}}^{\prime}, \mathbf{r}_{\mathbf{1}}, \mathbf{r}_{\mathbf{2}}\right)=\sum_{a b c d} \Gamma_{a b c d} \phi_{a}\left(\mathbf{r}_{1}^{\prime}\right) \phi_{b}\left(\mathbf{r}_{\mathbf{2}}^{\prime}\right) \phi_{c}\left(\mathbf{r}_{\mathbf{1}}\right) \phi_{d}\left(\mathbf{r}_{\mathbf{2}}\right)
$$

where $\Gamma_{a b c d}$ is a two-particle density matrix element. We note that, for Hartree-Fock (HF) wave functions, two-particle density matrix elements take the simple form

$$
\Gamma_{a b c d}^{\mathrm{HF}}=\frac{1}{2}\left(P_{a b}^{\alpha} P_{c d}^{\alpha}+P_{a b}^{\beta} P_{c d}^{\beta}-P_{a d}^{\alpha} P_{b c}^{\alpha}-P_{a d}^{\beta} P_{b c}^{\beta}\right)
$$

where $\mathbf{P}^{\alpha}$ and $\mathbf{P}^{\beta}$ are the HF alpha and beta density matrices.

Substituting Eq. (4) into Eq. (3) yields

$$
\Omega(u, v, \omega)=\sum_{a b c d} \Gamma_{a b c d}[a b c d]_{\Omega},
$$

where the Omega integrals

$$
[a b c d]_{\Omega}=\int \Phi_{a d}^{*}(\mathbf{u}, \mathbf{v}) \Phi_{b c}(\mathbf{u}, \mathbf{v}) \delta\left(\theta_{u v}-\omega\right) d \boldsymbol{\Omega}_{\mathbf{u}} d \mathbf{\Omega}_{\mathbf{v}}
$$

are formed from the phase functions

$$
\Phi_{a d}(\mathbf{u}, \mathbf{v})=(2 \pi)^{-3 / 2} \int \phi_{a}(\mathbf{r}) \phi_{d}(\mathbf{r}+\mathbf{u}) e^{i \mathbf{v} \cdot \mathbf{r}} d \mathbf{r}
$$

by integration over the angular parts of $\mathbf{u}$ and $\mathbf{v}$.

\section{B. The $[s s s s]_{\Omega}$ integral}

To progress further, we must specify the form of the basis functions. If one uses Gaussians, the resulting phase functions (8) can be found in closed form. For example, unnormalized $s$-type Gaussians, with exponents $\alpha, \beta, \gamma$, and $\delta$ on centers $\mathbf{A}, \mathbf{B}, \mathbf{C}$ and $\mathbf{D}$, yield

$$
\begin{aligned}
\Phi_{a d}(\mathbf{u}, \mathbf{v})= & (2 \alpha+2 \delta)^{-3 / 2} \exp \left[-\frac{\alpha \delta}{\alpha+\delta}|\mathbf{A}-\mathbf{D}+\mathbf{u}|^{2}\right. \\
& \left.-i \frac{\alpha \mathbf{A}+\delta \mathbf{D}-\delta \mathbf{u}}{\alpha+\delta} \cdot \mathbf{v}-\frac{v^{2}}{4(\alpha+\delta)}\right], \\
\Phi_{b c}(\mathbf{u}, \mathbf{v})= & (2 \beta+2 \gamma)^{-3 / 2} \exp \left[-\frac{\beta \gamma}{\beta+\gamma}|\mathbf{B}-\mathbf{C}+\mathbf{u}|^{2}\right. \\
& \left.-i \frac{\beta \mathbf{B}+\gamma \mathbf{C}-\gamma \mathbf{u}}{\beta+\gamma} \cdot \mathbf{v}-\frac{v^{2}}{4(\beta+\gamma)}\right],
\end{aligned}
$$

and, after introducing

$$
\begin{aligned}
& \lambda^{2}=\frac{\alpha \delta}{\alpha+\delta}+\frac{\beta \gamma}{\beta+\gamma}, \\
& 4 \mu^{2}=\frac{1}{\alpha+\delta}+\frac{1}{\beta+\gamma}, \\
& \eta=\frac{\alpha}{\alpha+\delta}-\frac{\beta}{\beta+\gamma}, \\
& \mathbf{P}=\frac{2 \alpha \delta}{\alpha+\delta}(\mathbf{A}-\mathbf{D})+\frac{2 \beta \gamma}{\beta+\gamma}(\mathbf{B}-\mathbf{C}), \\
& \mathbf{Q}=\frac{\alpha \mathbf{A}+\delta \mathbf{D}}{\alpha+\delta}-\frac{\beta \mathbf{B}+\gamma \mathbf{C}}{\beta+\gamma},
\end{aligned}
$$

$$
R=\frac{\alpha \delta}{\alpha+\delta}|\mathbf{A}-\mathbf{D}|^{2}+\frac{\beta \gamma}{\beta+\gamma}|\mathbf{B}-\mathbf{C}|^{2},
$$

the Omega integral (7) can be written as

$$
\begin{aligned}
{[\text { ssss }]_{\Omega}=} & \frac{\exp \left(-\lambda^{2} u^{2}-\mu^{2} v^{2}-i \eta u v \cos \omega-R\right)}{8(\alpha+\delta)^{3 / 2}(\beta+\gamma)^{3 / 2}} \\
& \times \int \exp (\mathbf{P} \cdot \mathbf{u}+i \mathbf{Q} \cdot \mathbf{v}) \delta\left(\theta_{u v}-\omega\right) d \mathbf{\Omega}_{\mathbf{u}} d \mathbf{\Omega}_{\mathbf{v}}
\end{aligned}
$$

This can be reduced ${ }^{8}$ to the one-dimensional integral,

$$
[\text { ssss }]_{\Omega}=C \exp (-R) \frac{1}{\pi} \int_{0}^{\pi} i_{0}(\sqrt{x+y \cos t}) d t
$$

where 


$$
\begin{aligned}
& C=\frac{\pi^{2} u^{2} v^{2} \sin \omega \exp \left(-\lambda^{2} u^{2}-\mu^{2} v^{2}-i \eta u v \cos \omega\right)}{(\alpha+\delta)^{3 / 2}(\beta+\gamma)^{3 / 2}}, \\
& x=(P u)^{2}+(i Q v)^{2}+2(P u)(i Q v) \cos \chi \cos \omega,
\end{aligned}
$$

$y=2(P u)(i Q v) \sin \chi \sin \omega$,

and $\mathbf{P} \cdot \mathbf{Q}=P Q \cos \chi$ and $i_{0}(x)=x^{-1} \sinh x$. The above $i_{0}$ integral can be accurately approximated using Gauss-Chebyshev quadrature. ${ }^{57}$ It can also be expressed as a infinite sum

$$
\begin{aligned}
{[\text { ssss }]_{\Omega}=} & C e^{-R} \sum_{n=0}^{\infty}(2 n+1) i^{n} i_{n}(P u) j_{n}(Q v) \\
& \times P_{n}(\cos \omega) P_{n}(\cos \chi),
\end{aligned}
$$

where $P_{n}(z)$ is the $n$th Legendre polynomial. This expression provides a possible alternative route to calculating $[\text { ssss }]_{\Omega}$ integrals.

\section{Higher $[a b c d]_{\Omega}$ integrals}

Following Boys, ${ }^{55}$ integrals of higher angular momentum can be formed by differentiating $[s s s s]_{\Omega}$ with respect to the Cartesian coordinates of the basis function centers. For example,

$$
\begin{aligned}
{\left[p_{z} s s s\right]_{\Omega}=\frac{1}{2 \alpha} \frac{\partial}{\partial A_{z}}[s s s s]_{\Omega}=} & \frac{C}{2 \alpha} \frac{\partial}{\partial A_{z}}\left[e^{-R} \frac{1}{\pi} \int_{0}^{\pi} i_{0}(\sqrt{x+y \cos t}) d t\right] \\
= & \frac{C}{2 \alpha}\left[\frac{1}{\pi} \int_{0}^{\pi} i_{0}(\sqrt{x+y \cos t}) d t \frac{\partial}{\partial A_{z}} e^{-R}+e^{-R} \frac{\partial}{\partial A_{z}} \frac{1}{\pi} \int_{0}^{\pi} i_{0}(\sqrt{x+y \cos t}) d t\right] \\
= & \frac{\delta}{\alpha+\delta}\left(D_{z}-A_{z}\right)[s s s s]_{\Omega}+\frac{C}{2} e^{-R}(\alpha+\delta)^{-1}\left[\left(2 u^{2} \delta P_{z}-v^{2} Q_{z}+i u v \cos \omega\left(P_{z}+2 \delta Q_{z}\right)\right) \frac{1}{\pi}\right. \\
& \times \int_{0}^{\pi} \frac{i_{1}(\sqrt{x+y \cos t})}{\sqrt{x+y \cos t}} d t+\left(Q_{z} \mathbf{P}-P_{z} \mathbf{Q}\right) \cdot(\mathbf{P}-2 \delta \mathbf{Q}) \frac{i u v \sin \omega}{P Q \sin \chi} \frac{1}{\pi} \int_{0}^{\pi} \frac{i_{1}(\sqrt{x+y \cos t})}{\sqrt{x+y \cos t} \cos t d t],}
\end{aligned}
$$

where $i_{1}(z)=z^{-1}\left(\cosh z-i_{0}(z)\right)$. It is straightforward, though very tedious, to form expressions in this way for integrals of higher angular momentum and we have derived and implemented up to $[p p p p]$ integrals within a development version of the Q-CHEM package. ${ }^{56}$ Once found, recurrence relations will be much more satisfactory.

\section{Bounds on $[a b c d]_{\Omega}$ integrals}

Because the total number of Omega integrals is $O\left(n^{4}\right)$, where $n$ is the number of basis functions, it is essential to be able to avoid the computation of negligible ones.

If $a$ and $b$ are real, then $\left|i_{0}(\sqrt{a+i b})\right|$ increases monotonically with $|b|$ and this yields the bound

$$
\left|[s s s s]_{\Omega}\right| \leqslant|C| \exp (-R)\left|i_{0}(\sqrt{x \pm y})\right|,
$$

where the sign is taken from the imaginary part of $x$. We will call Eq. (24) the four-center (4C) bound and it is obviously exact for all $[s s s]_{\Omega}$ with $y=0$. Unfortunately, although the bound turns out to be strong, its computational cost is not much smaller than that of $[\text { ssss }]_{\Omega}$ itself and it is thus of modest practical use.

Alternatively, applying the Cauchy-Schwarz inequality to Eq. (7) yields ${ }^{57}$

$$
\left|[a b c d]_{\Omega}\right| \leqslant B_{a d} B_{b c}
$$

where

$$
B_{a d}=\left[\int\left|\Phi_{a d}(\mathbf{u}, \mathbf{v})\right|^{2} \delta\left(\theta_{u v}-\omega\right) d \mathbf{\Omega}_{\mathbf{u}} d \mathbf{\Omega}_{\mathbf{v}}\right]^{1 / 2} .
$$

We will call Eq. (25) the Cauchy-Schwarz (CS) bound and it is obviously exact for all $[a b a b]_{\Omega}$ integrals. If both $\phi_{a}$ and $\phi_{d}$ are $s$-type Gaussians, one finds

$$
\begin{aligned}
B_{a d}= & \frac{\pi u v \sqrt{\sin \omega}}{(\alpha+\delta)^{3 / 2}} \exp \left[-\frac{\alpha \delta}{\alpha+\delta}\left(u^{2}+R_{A D}^{2}\right)-\frac{v^{2} / 4}{\alpha+\delta}\right] \\
& \times \sqrt{i_{0}\left(\frac{4 \alpha \delta}{\alpha+\delta} u R_{A D}\right)} \\
\leqslant & \frac{\pi u v \sqrt{\sin \omega}}{(\alpha+\delta)^{3 / 2}} \exp \left[-\frac{\alpha \delta}{\alpha+\delta}\left(u-R_{A D}\right)^{2}-\frac{v^{2} / 4}{\alpha+\delta}\right],
\end{aligned}
$$

where $R_{A D}=|\mathbf{A}-\mathbf{D}|$. Similar expressions can be found for basis functions of higher angular momentum.

Because it is a product of precomputable two-center quantities, the CS bound is valuable computationally. It is clear from Eq. (28), for example, that $[\text { ssss }]_{\Omega}$ is negligible 


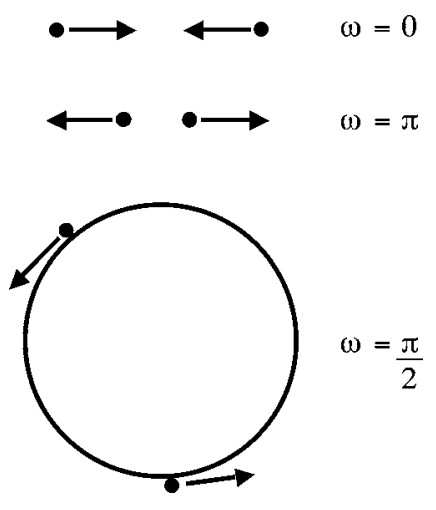

FIG. 1. Illustrations of three special values of $\omega$.

unless the internuclear distances $R_{A D}$ and $R_{B C}$ are both similar to $u$ and it follows that, in a large molecular system, there are only $O\left(n^{2}\right)$ significant Omega integrals. This is reminiscent of the analogous result ${ }^{58}$ for the more familiar Coulomb integrals $[a b \mid c d]$.

\section{E. The $\mathbf{Y}(\omega)$ intracule}

The Angle intracule

$$
\Upsilon(\omega)=\int_{0}^{\infty} \int_{0}^{\infty} \Omega(u, v, \omega) d u d v
$$

gives the distribution of $\omega$, the angle between the relative position vector, $\mathbf{r}_{12}=\mathbf{r}_{1}-\mathbf{r}_{2}$, and the relative momentum vector, $\mathbf{p}_{\mathbf{1 2}}=\mathbf{p}_{\mathbf{1}}-\mathbf{p}_{\mathbf{2}}$. Three special cases are shown in Fig. 1. In the first, where two electrons approach head on, we have $\omega$ $=0$. In the second, which is exactly the opposite, we have $\omega=\pi$. For geometric reasons, such collinear motions have vanishingly small probability densities in molecular systems and therefore $Y(0)=\Upsilon(\pi)=0$. In the third case, where the electrons follow a circular orbit at the same speed, it is easy to show that $\omega=\pi / 2$. These three cases suggest a way to interpret the Angle intracule: systems with electrons that tend to orbit one another have intracules that are enhanced around $\omega=\pi / 2$; systems with electrons that tend to move collinearly have intracules that are enhanced around $\omega=0$ and $\omega=\pi$.

\section{NUMERICAL TESTING}

\section{A. Bounds}

In order to test the $4 \mathrm{C}$ and $\mathrm{CS}$ bounds, we computed all the $[\text { ssss }]_{\Omega}$ integrals that arise from placing three Gaussian $s$ functions with exponents 6665.0, 21.06, and 0.1596 on the vertices of the graphitic substructure shown in Fig. $2\left(r_{\mathrm{CC}}\right.$

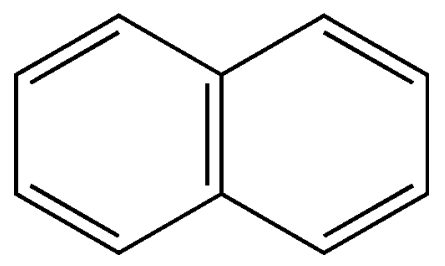

FIG. 2. Graphitic substructure used in bound testing.

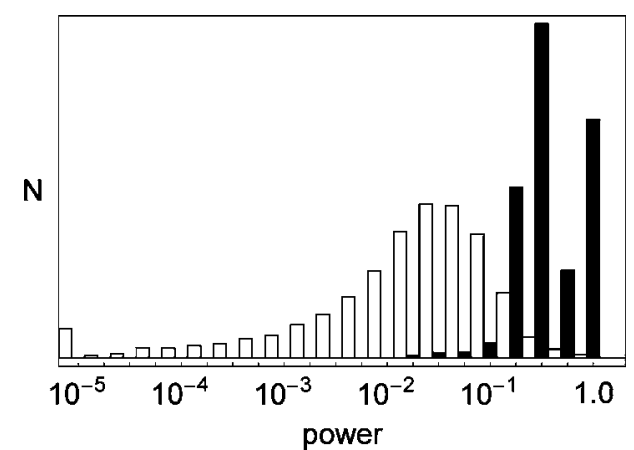

FIG. 3. Power profiles for 4C (black) and CS (white) bounds.

$=2.67963$ ). The exact value of each integral was computed by applying Gaussian quadrature to Eq. (18), using the adaptive Gauss-Kronrod algorithm ${ }^{59}$ as implemented in MATHEMATICA. ${ }^{60}$

Following Gill et al. ${ }^{61}$ we measure the effectiveness of each bound through the distribution of its power

$$
p=\frac{\mid[s s s]]_{\Omega} \mid}{\text { bound }}
$$

over the set of $[\text { ssss }]_{\Omega}$ integrals. A strong bound has a power profile close to unity; a weak bound has a profile close to zero. We arbitrarily selected the point $(u, v, \omega)=(4,4, \pi / 4)$ and expect that its profiles would be similar to those at other intermediate points. We define a negligible integral as having a value below $10^{-12}$ and report results only for nonnegligible integrals.

Power profiles for the $4 \mathrm{C}$ and CS bounds are illustrated in Fig. 3. The performance of these bounds, as measured by the the number of extra integrals calculated, is also summarized in Table I.

Figure 3 reveals that the $4 \mathrm{C}$ bound is approximately an order of magnitude stronger than the CS bound. Its superior performance is also reflected in Table I, which shows that the use of the CS bound leads to the calculation of $24 \%$ more integrals than are required while the use of the $4 \mathrm{C}$ bound leads to the calculation of only $6 \%$ more integrals than are required. Nonetheless, whereas the $4 \mathrm{C}$ bound must be evaluated $O\left(n^{4}\right)$ times, the CS bound is a product of two-center quantities and therefore needs to be evaluated only $O\left(n^{2}\right)$ times. Therefore, for maximum efficiency we use the CS bound to prescreen the integrals before invoking the $4 \mathrm{C}$ bound to make the final decision on which integrals to calculate.

TABLE I. The performance of the CS and $4 \mathrm{C}$ bounds, as measured by the number of extra non-negligible integrals as a percentage of the true number of non-negligible integrals.

\begin{tabular}{lc}
\hline \hline Bound & $\frac{N_{\text {extra }}}{N}(\%)$ \\
\hline None & 562 \\
CS bound & 24 \\
$4 \mathrm{C}$ bound & 6 \\
\hline
\end{tabular}


TABLE II. Values of the HF/6-311G Omega intracule for disilane using $n$-point Chebyshev quadrature.

\begin{tabular}{lccccccc}
\hline \hline$n$ & $\Omega(1,1, \pi / 2)$ & $\Omega(1,4, \pi / 2)$ & $\Omega(4,1, \pi / 2)$ & $\Omega(4,4, \pi / 2)$ & $\Omega(4,4, \pi / 4)$ & $\Omega(7,7, \pi / 2)$ & $\Omega(12,12, \pi / 2)$ \\
\hline 2 & 1.60980652983 & 5.69792417456 & 7.53915574157 & 5.90802631103 & 3.96373058001 & 0.56886331211 & 0.00002244838 \\
3 & 1.61023034347 & 5.69789492039 & 7.56147835087 & 5.90713927348 & 3.96681827806 & 0.56790134459 & 0.00002244924 \\
4 & 1.61022235251 & 5.69789253790 & 7.55923976761 & 5.90844380342 & 3.96665042804 & 0.56802499014 & 0.00002244923 \\
5 & 1.61022246736 & 5.69789251528 & 7.55929490139 & 5.90831247418 & 3.96666006698 & 0.56818220765 & 0.00002244923 \\
6 & 1.61022246665 & 5.69789251536 & 7.55929403024 & 5.90836142980 & 3.96665905256 & 0.56815121068 & 0.00002244923 \\
7 & 1.61022246665 & 5.69789251542 & 7.55929406224 & 5.90834742279 & 3.96665903006 & 0.56807908910 & 0.00002244923 \\
8 & 1.61022246665 & 5.69789251542 & 7.55929406105 & 5.90834876176 & 3.96665901990 & 0.56811589042 & 0.00002244923 \\
9 & 1.61022246665 & 5.69789251542 & 7.55929406108 & 5.90834879665 & 3.96665902626 & 0.56811019593 & 0.00002244923 \\
10 & 1.61022246665 & 5.69789251542 & 7.55929406108 & 5.90834881276 & 3.96665902519 & 0.56810967138 & 0.00002244923 \\
11 & 1.61022246665 & 5.69789251542 & 7.55929406108 & 5.90834879776 & 3.96665902523 & 0.56811006190 & 0.00002244923 \\
12 & 1.61022246665 & 5.69789251542 & 7.55929406108 & 5.90834880064 & 3.96665902525 & 0.56810998294 & 0.00002244923 \\
13 & 1.61022246665 & 5.69789251542 & 7.55929406108 & 5.90834880069 & 3.96665902524 & 0.56810999208 & 0.00002244923 \\
14 & 1.61022246665 & 5.69789251542 & 7.55929406108 & 5.90834880058 & 3.96665902524 & 0.56810998337 & 0.00002244923 \\
15 & 1.61022246665 & 5.69789251542 & 7.55929406108 & 5.90834880059 & 3.96665902524 & 0.56810999344 & 0.00002244923 \\
16 & 1.61022246665 & 5.69789251542 & 7.55929406108 & 5.90834880059 & 3.96665902524 & 0.56810998703 & 0.00002244923 \\
17 & 1.61022246665 & 5.69789251542 & 7.55929406108 & 5.90834880059 & 3.96665902524 & 0.56810998978 & 0.00002244923 \\
18 & 1.61022246665 & 5.69789251542 & 7.55929406108 & 5.90834880059 & 3.96665902524 & 0.56810998890 & 0.00002244923 \\
19 & 1.61022246665 & 5.69789251542 & 7.55929406108 & 5.90834880059 & 3.96665902524 & 0.56810998912 & 0.00002244923 \\
20 & 1.61022246665 & 5.69789251542 & 7.55929406108 & 5.90834880059 & 3.96665902524 & 0.56810998908 & 0.00002244923 \\
Exact & 1.61022246665 & 5.69789251542 & 7.55929406108 & 5.90834880059 & 3.96665902524 & 0.56810998908 & 0.00002244923 \\
\hline
\end{tabular}

\section{B. Chebyshev quadrature}

Although we have not been able to solve the integral (18) in closed form, it can be accurately approximated by Chebyshev quadrature ${ }^{57}$

$$
[s s s s]_{\Omega} \approx C e^{-R} \frac{1}{n} \sum_{j=1}^{n} i_{0}\left(\sqrt{x+y \cos \left(\left(j-\frac{1}{2}\right) \frac{\pi}{n}\right)}\right)
$$

and analogous quadratures are effective for the integrals over the higher $i_{n}$.

This quadrature approach was tested on the disilane $\left(\mathrm{Si}_{2} \mathrm{H}_{6}\right)$ molecule. The HF/6-311G wave function was calculated at the HF/6-311G optimized geometry $\left(r_{\mathrm{SiSi}}=4.42311\right.$, $\left.r_{\mathrm{SiH}}=2.79798, \theta_{\mathrm{SiSiH}}=110.362^{\circ}\right)$ with a self-consistent field convergence cutoff of $10^{-5}$, and the number $n$ of Chebyshev points was incrementally increased until the calculated intra- cule values converged to 11 decimal places. The results of these calculations, for selected points on the Omega intracule, are presented in Table II.

Convergence is rapid for small values of $u$ and $v$. Just six quadrature points are needed to obtain $\Omega(1,1, \pi / 2)$ to 11 decimal places and the $\Omega(1,4, \pi / 2)$ and $\Omega(4,1, \pi / 2)$ values also converge rapidly, requiring seven and nine points, respectively. The $\Omega(4,4, \pi / 2)$ and $\Omega(7,7, \pi / 2)$ values exhibit the slowest convergence, requiring 15 and 20 points, respectively. Although there is a trend toward slower convergence of the quadrature for higher values of $u$ and $v$, this does not pose a major problem in practice, for the magnitude of the intracule also declines sharply with increasing $u$ and $v$. As a result, fewer significant figures are required for convergence to a certain number of decimal places in this region, and fewer quadrature points are needed. This is illustrated by the

TABLE III. Optimal Mura parameters.

\begin{tabular}{|c|c|c|c|c|}
\hline & \multicolumn{2}{|c|}{$u$ quadrature } & \multicolumn{2}{|c|}{$v$ quadrature } \\
\hline & $n$ & $\alpha$ & $n$ & $\alpha$ \\
\hline $\mathrm{H}_{2}(R=0)$ & 21 & 5.0 & 31 & 20.0 \\
\hline $\mathrm{H}_{2}(R=1)$ & 21 & 5.0 & 31 & 20.0 \\
\hline $\mathrm{H}_{2}(R=2.5)$ & 21 & 5.0 & 31 & 20.0 \\
\hline $\mathrm{H}_{2}(R=3)$ & 31 & 5.0 & 31 & 20.0 \\
\hline $\mathrm{H}_{2}(R=4)$ & 31 & 5.0 & 31 & 20.0 \\
\hline $\mathrm{H}_{2}(R=10)$ & 51 & 10.0 & 31 & 20.0 \\
\hline $\mathrm{He}_{2}(R=10)$ & 101 & 10.0 & 31 & 20.0 \\
\hline $\mathrm{He}$ & 21 & 5.0 & 31 & 20.0 \\
\hline $\mathrm{Be}$ & 21 & 5.0 & 31 & 20.0 \\
\hline $\mathrm{CH}_{4}$ & 61 & 4.0 & 91 & 50.0 \\
\hline $\mathrm{NH}_{3}$ & 51 & 4.0 & 81 & 50.0 \\
\hline $\mathrm{H}_{2} \mathrm{O}$ & 51 & 4.0 & 81 & 50.0 \\
\hline $\mathrm{HF}$ & 51 & 4.0 & 81 & 50.0 \\
\hline $\mathrm{Ne}$ & 51 & 4.0 & 81 & 50.0 \\
\hline
\end{tabular}




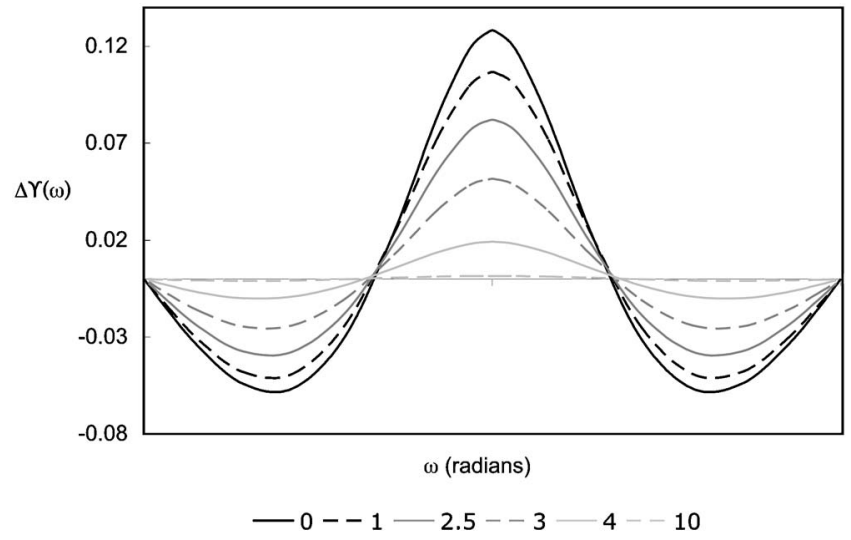

FIG. 4. $\Delta \mathrm{Y}(\omega)$ for $\mathrm{H}_{2}$ at $\mathrm{HF} / 6-311 \mathrm{G}^{* *}$, for various interatomic distances $R$ in a.u.

$\Omega(12,12, \pi / 2)$ case, where only four quadrature points are required to achieve convergence to 11 decimal places and seven significant figures. Finally, we observe that $\Omega(4,4, \pi / 4)$ and $\Omega(4,4, \pi / 2)$ require a similar number of points, suggesting that the convergence of the quadrature depends only weakly on $\omega$.

Overall, Chebyshev quadrature is well suited to evaluating $[a b c d]_{\Omega}$ integrals, requiring a maximum of 20 points to get convergence to 11 decimal places in the cases tested here. Moreover, if we are content with seven decimal places, a maximum of just 12 quadrature points is needed.

\section{The $Y(\omega)$ intracule}

The Angle intracule can be extracted from the Omega intracule by numerically integrating over the variables $u$ and $v$. In the present work, we have used the logarithmic quadrature grid of Mura and Knowles, ${ }^{62}$ enlarging each grid until the $\Upsilon(\omega)$ intracule converges to at least five decimal places. Optimal Mura parameters for the systems studied below are presented in Table III.

\section{RESULTS AND DISCUSSION}

\section{A. Dissociating $\mathrm{H}_{2}$}

The Angle intracule $Y(\omega)$ for two hydrogen atoms at infinite separation is simply

$$
\Upsilon_{\infty}(\omega)=\frac{\sin \omega}{2},
$$

reflecting a purely geometrical distribution of $\omega$ values. Angle intracules for $\mathrm{H}_{2}$ were computed from UHF/6-311G $\mathrm{G}^{* *}$ wave functions, at a range of internuclear distances, and the resulting differential intracules

$$
\Delta \Upsilon(\omega)=\Upsilon(\omega)-\Upsilon_{\infty}(\omega)
$$

are plotted in Fig. 4. It is clear that the intracule around $\omega=\pi / 2$ increases monotonically as the hydrogen molecule forms (and eventually fuses into a helium atom) and that this growth occurs at the expense of the regions close to $\omega=0$ and $\omega=\pi$. This reveals that, as the bond forms, the two electrons tend to orbit each other more.

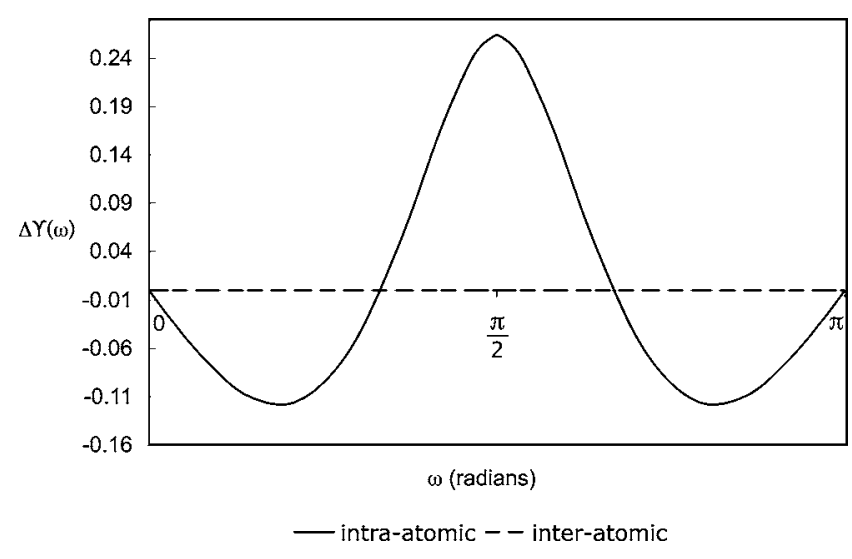

FIG. 5. $\Delta \Upsilon(\omega)$ for $\mathrm{He}_{2}(R=10$ a.u. $)$ at $\mathrm{HF} / 6-311 \mathrm{G}$.

\section{B. He...He and Be atom}

Angle intracules for the helium dimer $(R=10)$ and $\mathrm{Be}$ atom were calculated from HF/6-311G wave functions and differential intracules for these systems are shown in Figs. 5 and 6. Because the four-electron systems $\mathrm{He}_{2}$ and $\mathrm{Be}$ are more complicated than $\mathrm{H}_{2}$, we have partitioned their intracules into two-electron components. The $\mathrm{He}_{2}$ intracule was split into intra- and interatomic components, and the Be intracule into orbital-pair components. As in the $\mathrm{H}_{2}$ case above, our base line is an appropriate multiple of $Y_{\infty}(\omega)$.

Figure 5 reveals an enhancement near $\omega=\pi / 2$ for the intra-atomic intracule component, similar to that for the "fused" $\mathrm{H}_{2}$ case discussed above. As one might expect, the interatomic component is indistinguishable from $Y_{\infty}(\omega)$.

The orbital-pair components of the Be intracule, shown in Fig. 6, display more complex behavior. The $1 s / 1 s$ and $2 s / 2 s$ components show enhancement near $\omega=\pi / 2$, but the $1 s / 2 s$ curve is depleted in this region. The similarity of the $1 s / 1 s$ component to the intra-atomic intracule in Fig. 5 is not surprising but it is interesting that both the size and shape of the $2 s / 2 s$ component are significantly different. This suggests that the $2 s$ electrons deviate more from the simple circular model (Fig. 1) than the $1 \mathrm{~s}$ electrons, which, in turn, suggests that the position and momentum distributions of the $2 s$ electrons are more anisotropic than the $1 s$ ones.

The shape of the $1 s / 2 s$ component cannot be explained by the circular model, for the mean distances of the electrons

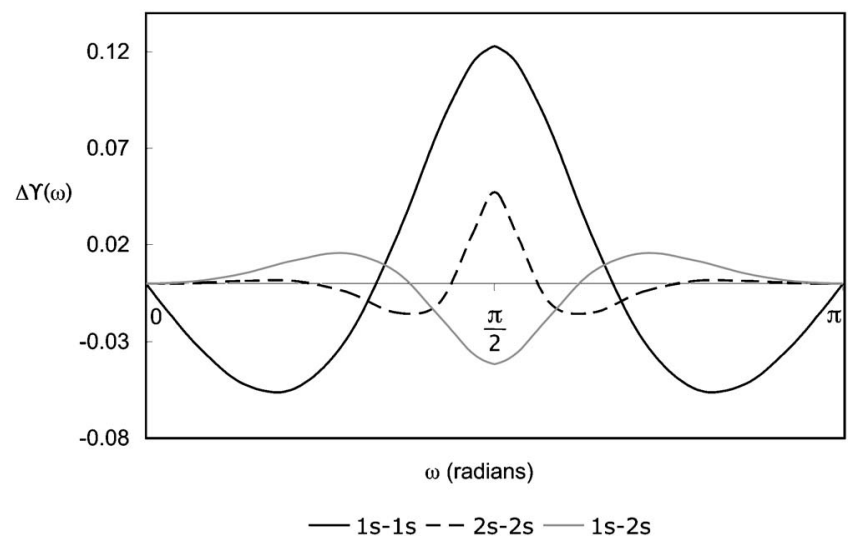

FIG. 6. Orbital-pair components to $\Delta \Upsilon(\omega)$ for the Be atom at HF/6-311G. 


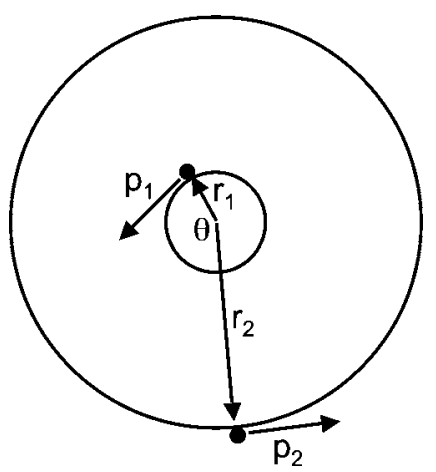

FIG. 7. Two particles orbiting a common center.

from the nucleus are no longer equal. However, if the electrons are assumed instead to move in orbits with different radii and momenta (Fig. 7), it is easy to show

$$
\omega=\cos ^{-1}\left[\frac{\left(r_{1} p_{2}-r_{2} p_{1}\right) \sin \theta}{r_{12} p_{12}}\right],
$$

and, if $p_{1}=p_{2}$, this reduces to

$$
\omega=\cos ^{-1}\left[\frac{\left(r_{2}-r_{1}\right) \cos (\theta / 2)}{r_{12}}\right] .
$$

We see that, as $\theta$ varies, $\omega$ takes all values between 0 and $\pi / 2$ and we conclude that radial inequivalence of the two electrons leads to a depletion near $\omega=\pi / 2$. This is likely to be responsible for the shape of the $1 s / 2 s$ component.

As the intracule for any atomic or molecular system can be expressed in terms of shell-pair components, we expect that the intracules of systems with a greater number of intershell electron pairs will be relatively depleted about $\pi / 2$, while the intracules of systems with a greater number of intrashell electron pairs will be relatively enhanced about $\pi / 2$.

\section{Isoelectronic molecular species}

The Angle intracules of $\mathrm{CH}_{4}\left(r_{\mathrm{CH}}=2.05\right), \mathrm{NH}_{3}\left(r_{\mathrm{NH}}\right.$ $\left.=1.91, \theta_{\mathrm{HNH}}=107.0^{\circ}\right), \mathrm{H}_{2} \mathrm{O}\left(r_{\mathrm{OH}}=1.81, \theta_{\mathrm{HOH}}=104.4^{\circ}\right), \mathrm{HF}$ $\left(r_{\mathrm{HF}}=1.73\right)$, and $\mathrm{Ne}$ were calculated from their HF/6-311G wave functions, and $\Delta \Upsilon(\omega)$ plots relative to the $\mathrm{Ne}$ atom are

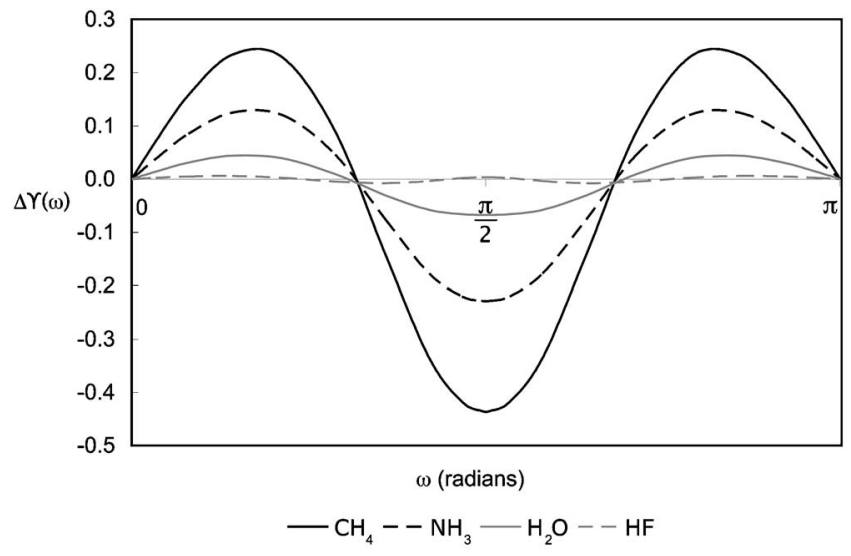

FIG. 8. $\Delta \mathrm{Y}(\omega)$ for the $\mathrm{CH}_{4}, \mathrm{NH}_{3}, \mathrm{H}_{2} \mathrm{O}$, and HF molecules, relative to the $\mathrm{Ne}$ atom, at HF/6-311G.

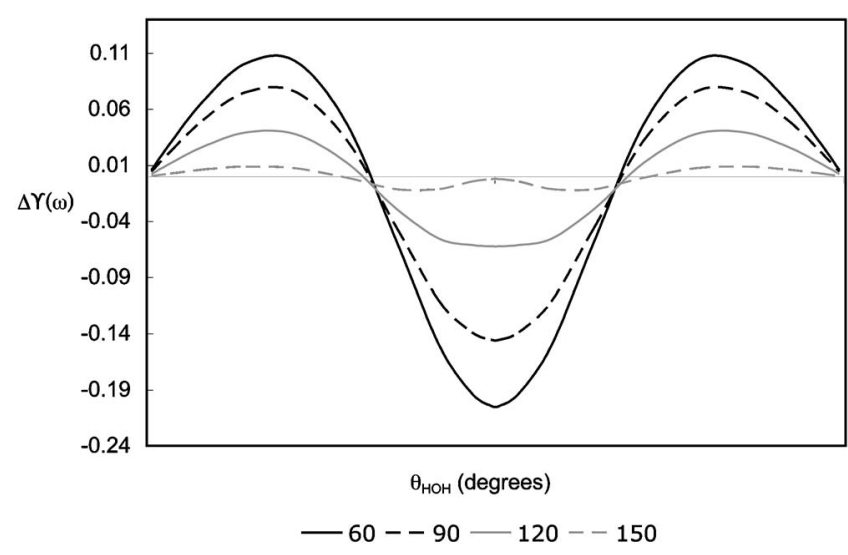

FIG. 9. $\Delta \Upsilon(\omega)$ for water at HF/6-311G, for various values of the internal angle $\theta_{\mathrm{HOH}}$ in degrees.

shown in Fig. 8. As we move from $\mathrm{Ne}$ to $\mathrm{CH}_{4}$, the intracule becomes progressively more depleted near $\omega=\pi / 2$ and enhanced near $\omega=0$ and $\omega=\pi$. This is reasonable, suggesting that some of the circular motion of electron pairs in $\mathrm{Ne}$ (a spherical system) is replaced by collinear motion in $\mathrm{CH}_{4}$ (a system with four $\sigma$ bonds). The molecular species $\mathrm{HF}, \mathrm{H}_{2} \mathrm{O}$, and $\mathrm{NH}_{3}$ lie between these two extremes, and their intracules vary according to the number of $\sigma$ bonds that they contain.

\section{Geometry dependence}

Angle intracules for the angle-bending mode of water $\left(r_{\mathrm{OH}}=1.81\right)$ and the umbrella mode of ammonia $\left(r_{\mathrm{NH}}\right.$ $=1.91$ ) were calculated from HF/6-311G wave functions, and their differential intracules

$$
\Delta \Upsilon(\omega)=\Upsilon(\omega)-\Upsilon_{\text {planar }(\omega)}
$$

are plotted in Figs. 9 and 10. In both cases, we observe depletion of the intracule about $\omega=\pi / 2$ and enhancement near $\omega=0$ and $\omega=\pi$ as the molecule deviates from planarity, suggesting that the planar molecule is somehow "more spherical" than its nonplanar counterparts. As the nature of the $\mathrm{O}-\mathrm{H}$ and $\mathrm{N}-\mathrm{H}$ sigma bonds is not expected to change greatly with molecular distortion, we suspect that it is the behavior of the lone pair electrons that is the major factor contributing to the change in the intracule. Specifically, we

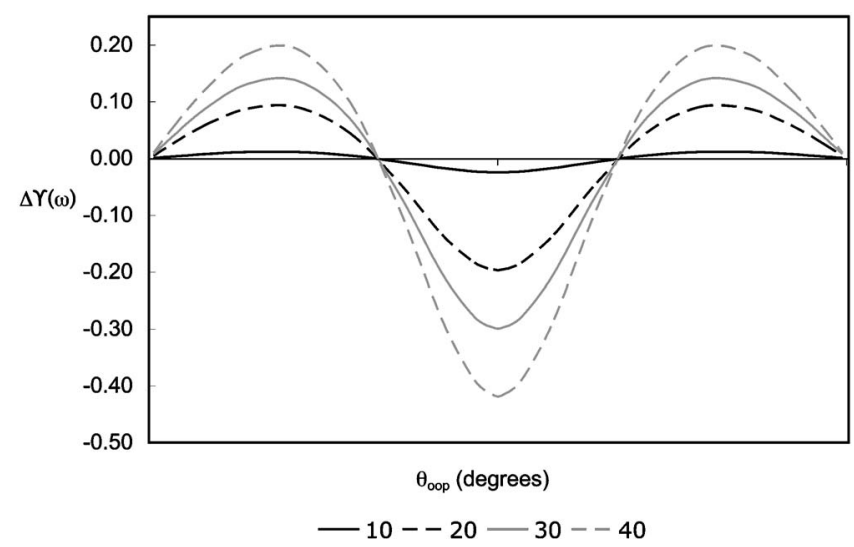

FIG. 10. $\Delta \mathrm{Y}(\omega)$ for ammonia at HF/6-311G, for various out-of-plane dihedral angles $\theta_{\text {oop }}$ in degrees. 


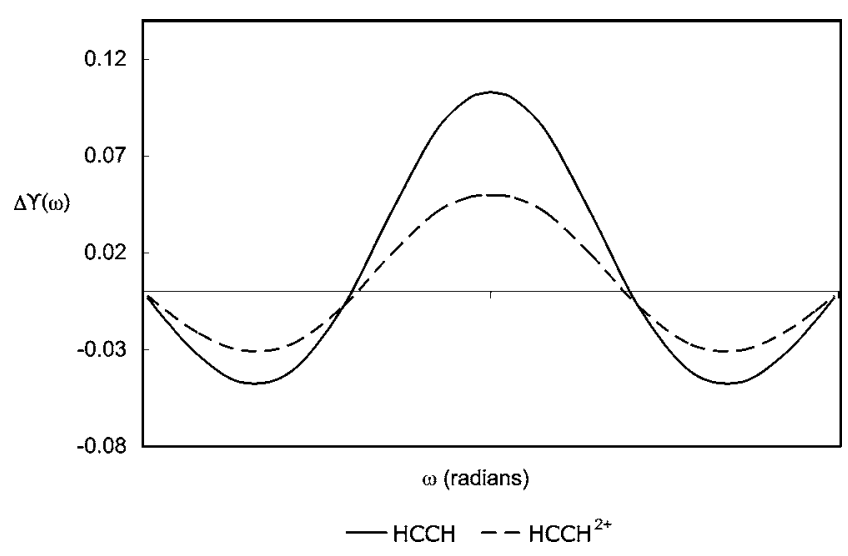

FIG. 11. Normalized $\Delta \mathrm{Y}(\omega)$ for ethyne and ethyne ${ }^{2+}$, relative to ethyne ${ }^{4+}$, at $\mathrm{HF} / 6-311 \mathrm{G}$

attribute the change in the intracule to a change in the localization of the lone pair electrons, which are free to orbit the linear water and planar ammonia conformers, but get confined to spatially localized orbitals in the bent water and nonplanar ammonia conformers. This result suggests that systems with greater electron delocalization, such as $\pi$-bonded molecules, will have stronger "orbiting" character than their $\sigma$-bonded counterparts. This conjecture will be explored in the following section.

\section{E. $\sigma$-versus $\pi$ bonding}

The Angle intracules of $\mathrm{HCCH}, \mathrm{HCCH}^{2+}$, and $\mathrm{HCCH}^{4+}$ $\left(r_{\mathrm{CC}}=2.27, r_{\mathrm{CH}}=2.01\right)$ were calculated from their HF/6-311G wave functions, then normalized by the number of electron pairs. The normalized $\mathrm{HCCH}^{4+}$ intracule was used as a base line for the $\Delta \Upsilon(\omega)$ plots shown in Fig. 11. From this figure, we observe that as the $\pi$ orbitals become occupied (moving from $\mathrm{HCCH}^{4+}$ to $\mathrm{HCCH}^{2+}$ to $\mathrm{HCCH}$ ), the intracule builds up around $\omega=\pi / 2$ and depletes near $\omega=0$ and $\omega=\pi$. This is entirely consistent with our previous observations that systems with delocalized electrons are more spherical than highly localized systems, and that this can be quantified as an enhancement of the Angle intracule about $\pi / 2$.

\section{CONCLUSIONS}

The Omega intracule $\Omega(u, v, \omega)$ is a rich function that distills key physical information from the complexity of a full multidimensional electronic wave function. It captures critical information about the relative positions $u$ and momenta $v$ of the electrons and these play a major role in understanding electron correlation effects. The distribution of the angle $\omega$ appears to reflect the nature of the bonding in the system and may be useful in modeling subtler correlation effects. We are exploring this possibility.

\section{ACKNOWLEDGMENTS}

The authors are grateful to Dr. Élise Dumont for stimulating discussions. We also thank the ANU and ARC (Grant Nos. DP0664466 and DP0771978) for funding.
${ }^{1}$ P. Hohenberg and W. Kohn, Phys. Rev. B 136, 864 (1964).

${ }^{2}$ A. M. Lee and P. M. W. Gill, Chem. Phys. Lett. 313, 271 (1999).

${ }^{3}$ P. M. W. Gill, A. M. Lee, N. Nair, and R. D. Adamson, J. Mol. Struct.: THEOCHEM 506, 303 (2000).

${ }^{4}$ N. A. Besley, A. M. Lee, and P. M. W. Gill, Mol. Phys. 100, 1763 (2002).

${ }^{5}$ N. A. Besley, D. P. O’Neill, and P. M. W. Gill, J. Chem. Phys. 118, 2033 (2003).

${ }^{6}$ P. M. W. Gill, D. P. O'Neill, and N. A. Besley, Theor. Chem. Acc. 109, $241(2003)$

${ }^{7}$ D. P. O'Neill and P. M. W. Gill, Phys. Rev. A 68, 022505 (2003).

${ }^{8}$ P. M. W. Gill, D. L. Crittenden, D. P. O'Neill, and N. A. Besley, Phys. Chem. Chem. Phys. 8, 15 (2006).

${ }^{9}$ N. A. Besley, J. Chem. Phys. 125, 074104 (2006).

${ }^{10}$ N. A. Besley, Chem. Phys. Lett. 409, 63 (2005).

${ }^{11}$ A. S. Eddington, Fundamental Theory (Cambridge, England, 1946).

${ }^{12}$ C. A. Coulson and A. H. Neilson, Proc. Phys. Soc. London 78, 931 (1961).

${ }^{13}$ W. A. Lester and M. Krauss, J. Chem. Phys. 41, 1407 (1964).

${ }^{14}$ R. F. Curl and C. A. Coulson, Proc. Phys. Soc. London 85, 647 (1965).

${ }^{15}$ A. J. Coleman, Int. J. Quantum Chem., Symp. 1S, 457 (1967).

${ }^{16}$ K. E. Banyard and C. C. Baker, J. Chem. Phys. 51, 2680 (1969).

${ }^{17}$ R. Benesch and V. H. Smith, Acta Crystallogr., Sect. A: Cryst. Phys., Diffr., Theor. Gen. Crystallogr. 26, 579 (1970).

${ }^{18}$ R. Benesch and V. H. Smith, J. Chem. Phys. 55, 482 (1971).

${ }^{19}$ R. J. Boyd and C. A. Coulson, J. Phys. B 6, 782 (1973).

${ }^{20}$ A. J. Thakkar and V. H. Smith, Chem. Phys. Lett. 42, 476 (1976).

${ }^{21}$ A. J. Thakkar and V. H. Smith, J. Chem. Phys. 67, 1191 (1977).

${ }^{22}$ K. E. Banyard and C. E. Reed, J. Phys. B 11, 2957 (1978).

${ }^{23}$ R. J. Boyd and M. C. Yee, J. Chem. Phys. 77, 3578 (1982).

${ }^{24}$ A. J. Thakkar, A. N. Tripathi, and V. H. Smith, Int. J. Quantum Chem. 26, 157 (1984).

${ }^{25}$ P. E. Regier and A. J. Thakkar, J. Phys. B 18, 3061 (1985).

${ }^{26}$ J. M. Ugalde and R. J. Boyd, Int. J. Quantum Chem. 29, 1 (1986).

${ }^{27}$ R. J. Boyd, C. Sarasola, and J. M. Ugalde, J. Phys. B 21, 2555 (1988).

${ }^{28}$ K. E. Banyard, K. H. Albayati, and P. K. Youngman, J. Phys. B 21, 3177 (1988).

${ }^{29}$ C. Sarasola, J. M. Ugalde, and R. J. Boyd, J. Phys. B 23, 1095 (1990).

${ }^{30}$ J. M. Ugalde, C. Sarasola, L. Dominguez, and R. J. Boyd, J. Math. Chem. 6, 51 (1991)

${ }^{31}$ J. Wang, A. J. Thakkar, and V. H. Smith, J. Chem. Phys. 97, 9188 (1992).

${ }^{32}$ T. Koga, Y. Kasai, and A. J. Thakkar, Int. J. Quantum Chem. 46, 689 (1993).

${ }^{33}$ J. Wang and V. H. Smith, Int. J. Quantum Chem. 49, 147 (1994).

${ }^{34}$ J. Wang and V. H. Smith, Chem. Phys. Lett. 220, 331 (1994).

${ }^{35}$ J. Wang and V. H. Smith, J. Phys. B 27, 5159 (1994).

${ }^{36}$ J. Cioslowski, B. B. Stefanov, A. Tan, and C. J. Umrigar, J. Chem. Phys. 103, 6093 (1995).

${ }^{37}$ J. Cioslowski and G. H. Liu, J. Chem. Phys. 105, 4151 (1996).

${ }^{38}$ J. Cioslowski and G. H. Liu, J. Chem. Phys. 105, 8187 (1996).

${ }^{39}$ J. Cioslowski and G. H. Liu, J. Chem. Phys. 109, 8225 (1998).

${ }^{40}$ A. Sarsa, F. J. Galvez, and E. Buendia, J. Chem. Phys. 109, 7075 (1998).

${ }^{41}$ H. Matsuyama, T. Koga, E. Romera, and J. S. Dehesa, Phys. Rev. A 57, 1759 (1998)

${ }^{42}$ T. Koga and H. Matsuyama, J. Phys. B 31, 3765 (1998).

${ }^{43}$ X. Fradera, C. Sarasola, J. M. Ugalde, and R. J. Boyd, Chem. Phys. Lett. 304, 393 (1999).

${ }^{44}$ A. Sarsa, F. J. Galvez, and E. Buendia, J. Chem. Phys. 110, 5721 (1999).

${ }^{45}$ F. A. de Saavedra, E. Buendia, F. J. Galvez, and A. Sarsa, Int. J. Quantum Chem. 79, 75 (2000).

${ }^{46}$ E. Valderrama, X. Fradera, and J. M. Ugalde, J. Chem. Phys. 115, 1987 (2001).

${ }^{47}$ A. J. Thakkar and B. S. Sharma, Int. J. Quantum Chem. 85, 258 (2001).

${ }^{48}$ T. Koga, J. Chem. Phys. 116, 6910 (2002).

${ }^{49}$ T. Koga, J. Chem. Phys. 117, 10493 (2002).

${ }^{50}$ T. Koga, Theor. Chem. Acc. 110, 79 (2003)

${ }^{51}$ E. G. Valderrama and J. M. Ugalde, J. Math. Chem. 37, 211 (2005).

${ }^{52}$ R. Fondermann, M. Hanrath, M. Dolg, and D. P. O'Neill, Chem. Phys. Lett. 413, 237 (2005).

${ }^{53}$ E. Wigner, Phys. Rev. 40, 749 (1932).

${ }^{54}$ R. G. Parr and W. Yang, Density Functional Theory of Atoms and Molecules (Oxford University Press, New York, 1989). 
${ }^{55}$ M. Abramowitz and I. A. Stegun, Handbook of Mathematical Functions with Formulas, Graphs, and Mathematical Tables (Dover, New York, 1964).

${ }^{56}$ Y. Shao, L. Fusti-Molnar, and Y. Jung et al., Phys. Chem. Chem. Phys. 8 $3172(2006)$

${ }^{57}$ S. F. Boys, Proc. R. Soc. London, Ser. A 200, 542 (1950).
${ }^{58}$ M. Haser and R. Ahlrichs, J. Comput. Chem. 10, 104 (1988).

${ }^{59}$ A. S. Kronrod, Dokl. Akad. Nauk SSSR 154, 283 (1964).

${ }^{60}$ MATHEMATICA, Version 5, Wolfram Research Inc., Champaign, IL, 2003).

${ }^{61}$ P. M. W. Gill, B. G. Johnson, and J. A. Pople, Chem. Phys. Lett. 217, 65 (1994).

${ }^{62}$ M. E. Mura and P. J. Knowles, J. Chem. Phys. 104, 9848 (1996). 